\title{
Fast Recursive Algorithm and Application of a Multichannel Correlation Coefficient Formula
}

\author{
Chen Huamin, Cao yuan \\ Nanyang Institute of Technology, Nanyang 473004, China \\ chm2422@163.com
}

Keywords: signal energy, multichannel, correlation, weak signal detection

Abstract: Coherence technology is a kind of method which uses mathematic methods to highlight the similarity of the signal between two neighbouring channels in order to detect weak signal and reflect unusual characters. Fast recurring algorithm and application in weak signal detection of a multichannel correlation coefficient formula based on signal energy was introduced in this paper. This paper developed a set of application software of coherence technology based on the discussion about the realizing method of the mathematic model. The tests on theoretic model and practical model proved the availability of the algorithm.

\section{Introduction}

Weak signal detection is a new arisen course, the application of which covers various kinds of fields. Weak signal detection uses the methods of electronics, information theory, computer and physics to analyze the cause and law of noises, to study the character and coherence character of the signal and measure the useful signal which is submerged by noises ${ }^{[1]}$. The purpose of weak signal detection is to collect useful signal from strong noises or adopt some new technology and methods to increase signal-to-noise ratio (SNR) of the output signal ${ }^{[2]}$. The study on weak signal detection theory, seeking new methods and developing new equipment of weak signal detection are the central issues in measurement technology field.

The interference of noises is the main problem of information science. Extracting weak sine periodic signal is a synthetically technology and a advanced branch of signal detection. The devices of measuring amplitude and angle are widely used in various kinds of scientific fields such as physics, bio-medical science, earthquake etc ${ }^{[3]}$. At present, methods in time domain have the shortcomings of high door limitation and weak resistance to noises. But coherence technology hasn't these shortcomings apart from many strong points.

Coherence technology is a kind of method which uses mathematic methods to highlight the similarity of the signal between two neighbouring channels in order to detect weak signal and reflect unusual characters. In technique aspect, more and more attention is paid on coherence technology which is divided into three generations in the world. The algorithm of the first generation is based on correlation which has good signal distinguishing ability when the data has high signal-to-noise ratio but has weak resistance to noises ${ }^{[4]}$. The algorithm of the second generation is in view of similarity which is a better algorithm of calculating coherence character and 
has higher distinguishing ability compared with the first generation algorithm ${ }^{[5]}$. But the quality of the data still has effect on calculating coherence to some extent ${ }^{[6,7]}$. The third generation algorithm is based on character structure which is the highest level of coherence technology. In recent years, coherence technology developed quickly ${ }^{[8]}$. The author developed a set of application software of coherence technology based on the discussion about the realizing method of the mathematic model. The tests on theoretic model and practical model show that this method is effective in weak signal detection.

\section{Principle of 2-D Coherence Technology}

The principle of multichannel correlation coefficient formula based on signal energy is as following: firstly calculating the similarity coefficient of the vertical line and the horizontal line from a point of the space, then combining the calculated value to get the coherence value of the point. Secondly calculating the coherence value of each point along the time direction. Finally repeating this process to obtain the whole coherence body. Much time is needed according to this algorithm, therefore, the formula of multiple channels coherence coefficient and a faster algorithm started with energy of the signal is introduced.

Assuming a signal as $X_{j}(n), j=1,2, \ldots, M, n=1,2, \ldots, ; N ; M$ is the total number of the channels; $N$ is the number of points in the calculating window. Assuming a standard channel $\overline{X(n)}$, then the energy error between the standard channel and the Mth channel is $Q, Q=\sum_{j=1}^{M} \sum_{n=1}^{N}\left[X_{j}(n)-\overline{X(n)}\right]^{2}$, . To make $\frac{\partial Q}{\partial \overline{X(n)}}=0$, so that $Q$ can reach the minimum value. At this moment $\overline{X(n)}=\frac{1}{M} \sum_{J=1}^{M} X_{j}(n)$. According to the energy relationship between the standard channel and the $M$ th channel, the coherence value is got.

$$
R=\frac{\sum_{n=1}^{N}\left[\sum_{J=1}^{M} X_{j}(n)\right]^{2}-\sum_{j=1}^{M} \sum_{n=1}^{N} X_{j}^{2}(n)}{(M-1) \sum_{j=1}^{M} \sum_{n=1}^{N} X_{j}^{2}(n)}
$$

Bigger value of $R$ indicates better similarity character of $M$ channels' signal, otherwise, the similarity character is worse. The calculating amount of this method is great and the calculating speed is slow.

To change the cycling sequence of calculating, that is to calculate along to the horizontal line firstly, then to calculate along the vertical direction. Assuming the starting point and the terminal point of the glide window is $i$ and $N$, then they will change to $i+1$ and $N+1$ after the window gliding one point. I and II can be successively deduced and calculated individually, $i$ is the sequence number of the glide window whose original value is 1 . Then formula (1) can be written as:

$$
\begin{aligned}
& \mathrm{I}=\mathrm{I}+\left[\sum_{j} X_{j}(N+1)\right]^{2}-\left[\sum_{j} X_{j}(i)\right]^{2} \\
& \mathrm{II}=\mathrm{II}+\sum_{j}\left[X_{j}^{2}(N+1)-X_{j}^{2}(i)\right]
\end{aligned}
$$

From the formula above, the conclusion is reached. When calculating the coherence value of the 
next window, only to calculate the energy change of the two rows outside the overlapping part of the two windows and it is not necessary to calculate the energy of $N$ points inside the window. The calculating amount of this method is reduced according to geometry progression and the calculating speed is fast.

\section{Tests on Theoretic Model}

The procedure of this method is composed and tests on the theoretic model are done on the basis of analyzing the formula above. The result of the tests accords with the theoretic result. In the tests, the total number of the channels of the data is 24 and the number of the points in each channel is 1024 which is synthesized by half a period's sine signal and random signal. As figure 1 to figure 4 shows.
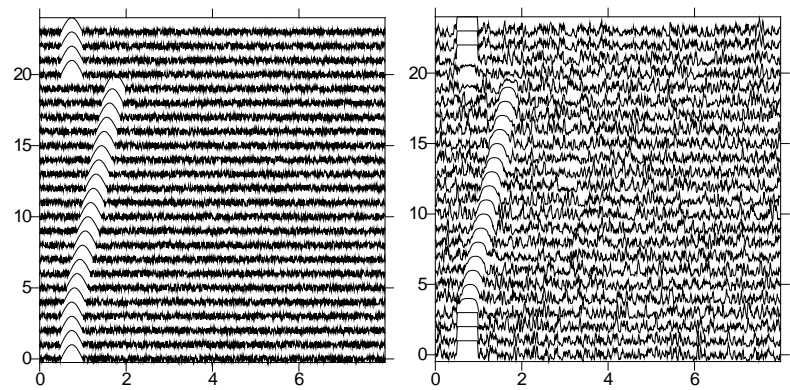

Fig.1. The input signal with $S / N$ being 0.5 (left) and the coherence figure (right)(glide window is $3 \times 5$ )

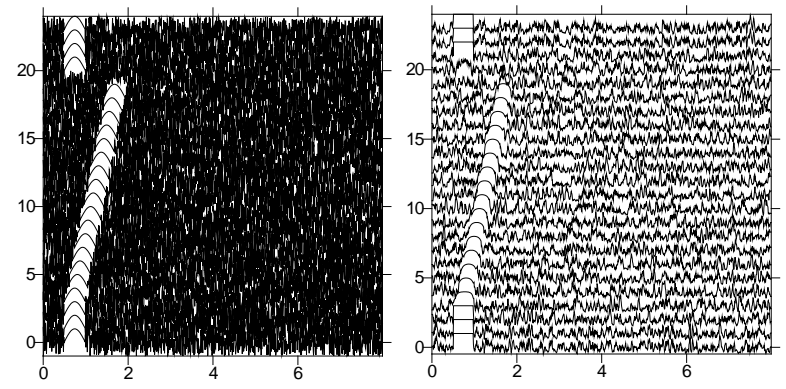

Fig.2. The input signal with $S / N$ being 0.5 (left) and the coherence figure (right)(glide window is $3 \times 5$ )

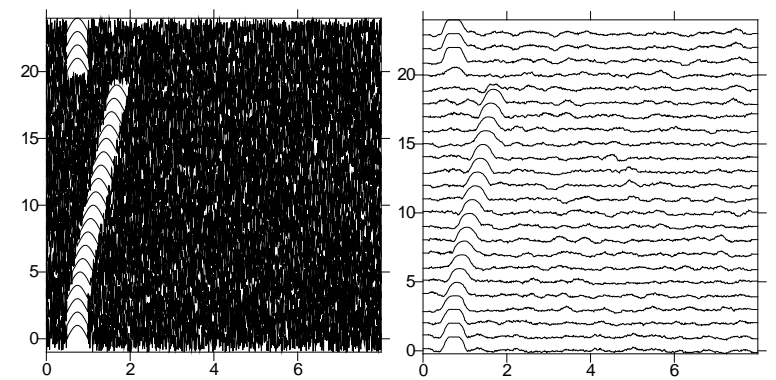

Fig.3. The input signal with $S / N$ being 1 (left) and the coherence figure (right)(glide window is $3 \times 33$ ) 

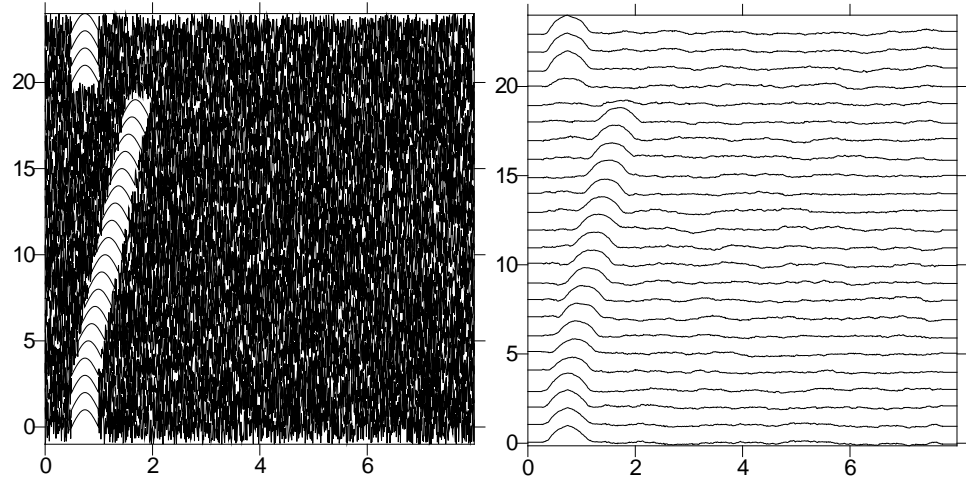

Fig.4. The input signal with $S / N$ being 1 (left) and the coherence figure (right)(glide window is $3 \times 65$ )

Through the analysis of figure 1 to figure 4, the conclusion is obtained. The character in the input signal is obviously reflected in the output signal. In order to show the comparison more directly, the output data is filled so that the number of the total channels and points in the input signal is completely equal to that of the output signal.

Comparing the input wave and the output wave, the result is clear.

(1) The completely same part of the 1st channel to the 4th channel in the input wave is reflected as a horizontal line in the 2nd channel to the 3rd channel in the output wave accordingly which means strong coherence character, as figure 1 and figure 2 show.

(2) If the length of the glide window is larger than the period of the input sine signal, then the outline and the period of the signal can't be accurately showed in the coherence wave. Generally speaking, the period in the coherence wave is longer than that of the original signal.

(3) The size of the calculating glide window must ensure enough data in it because it has much influence on coherence calculating. Too large glide window will bring smoothing effect to the coherence value, greatly suppressing random noises and smoothing a part of weak signal which is not benefit to detect weak signal; small glide window will sensitively check the small change of the wave which will enhance the resolving power to weak signal and accurately reflect weak signal but will increase the noises at the same time as figure 3 and figure 4 show. The length of the glide window in figure 4 is two times of that in figure 3, so the amplitude of valid signal wave is clearly smaller than that of figure 3.

(4) The relay in the input wave is also showed in the output wave. In the input wave the 5th channel to the 20th channel individually has one eighth period's relay than the former channel. Accordingly in the output wave the 4th channel to the 19th channel also individually has a fixed relay than the former channel as figure 1 to figure 4 show.

(5) When the signal-to-noise ratio (SNR) is 1:1, the signal is a kind of weak signal, however, the signal still can be obviously distinguished in the coherence figure as figure 2 to figure 4 show.

\section{Tests on the Practical model}

In order to further verify the validity of this method, examinations on practical model are done. The result shows that this method is effective in practical problems as figure 5 and figure 6 show. Figures below show that the results of practical model accord with that of the theoretic model.

Through the tests on theoretic model and practical model the weak signal detection ability of this method is verified. 

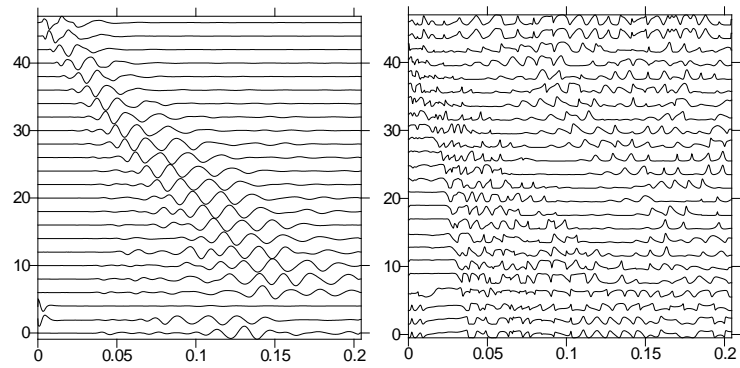

Fig .5. Practical data (left) and the coherence figure (right)(glide window is $3 \times 5$ )
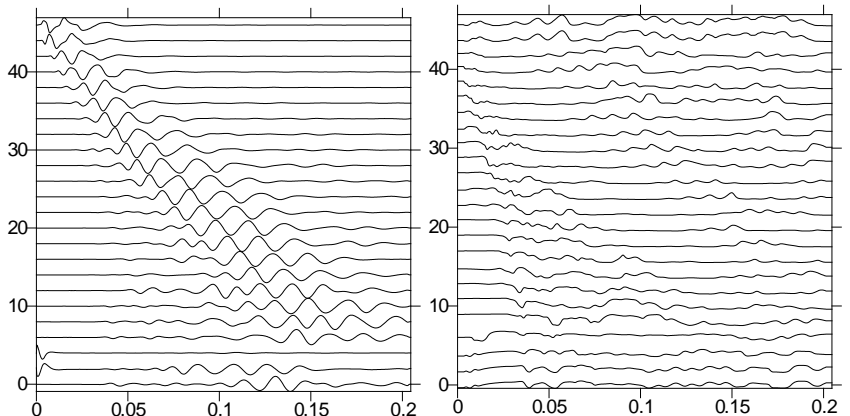

Fig.6 Practical data (left) and the coherence figure (right)(glide window is $3 \times 33$ )

\section{Conclusion}

The coherence analysis between two signals can show the extent of their similarity. In sound emitting, in order to detect, distinguish and extract one or more relayed signal, the coherence technology is indispensable. Just as spectrum analysis in frequency field, the coherence analysis in time domain has wide application in signal treatment field.

Considering this method in industrial practical application, the coherence technology in weak signal detection can be used to measure pipeline leaking and realize on-line monitoring of pipeline. With the improvement of the technology, the expanding of the application area, weak signal detection theory is continuously updated and the methods are constantly improved and increased. In practical application, different methods should be used in different actual conditions.

Weak signal detection has close relationships with many fields whose development is limited by solid ware conditions before. The maturing of weak signal detection technology will bring revolutionary development to this field before long in the future.

\section{References}

[1] József Simon, Exploring the changes in a series of measurements - The comparison of the two-dimensional correlation analysis and the alteration analysis[J], Chemometrics and Intelligent Laboratory Systems, 2017, pp.28-37

[2] Wang Yan. Ascertaining the Optimal Quantitative Wave number Range of Gas with Two-Dimensional Correlation Infrared Spectroscopy [J], Spectroscopy and Spectral Analysis, 2009, Vol.29, pp.:1835-1839

[3] Li Nan, Three nonlinear methods of weak signal detection[J], Electric Power Automation Equipment, 2008, Vol. 28, pp.: 82-85. 